\title{
New records of millipedes from the Comoro Islands (Diplopoda)
}

\section{Новые находки многоножек-диплопод с Коморских островов (Diplopoda)}

\section{Rollard ${ }^{1} \&$ S.I. Golovatch ${ }^{2}$ К. Ромлар ${ }^{1}$, С.И. Головач ${ }^{2}$}

\footnotetext{
${ }^{1}$ Muséum national d'Histoire naturelle, Département Systématique \& Evolution, UMR 7205 OSEB, Section Arthropodes, 57 rue Cuvier, CP 53, 75005 Paris, France.

${ }^{2}$ Institute for Problems of Ecology and Evolution, Russian Academy of Sciences, Leninsky pr. 33, Moscow 119071, Russia.

${ }^{2}$ Институт проблем экологии и эволюции РАН, Ленинский пр. 33, Москва 119071 Россия.
}

KEY WORDS: Diplopoda, fauna, Comoros.

КЛЮЧЕВЫЕ СЛОВА: Diplopoda, фауна, Коморские острова.

ABSTRACT. A small collection of diplopods from the Comoros contains nine species, two of which represent new island records. The fauna (16 species) is confirmed to be depauperate and dominated by anthropochore species, but several seem to be local endemics (4) or subendemics (3).

РЕЗЮМЕ. Небольшой материал диплопод с Коморских островов содержит девять видов, два из которых впервые указаны для одного из островов. Подтверждается, что фауна (16 видов) обеднена и в ней доминируют антропохорные виды, но в ее составе, очевидно, есть и местные эндемики (4) и субэндемики (3).

\section{Introduction}

The millipede fauna of the Comoros has recently been reviewed and shown to contain 16 species, all keyed [VandenSpiegel \& Golovatch, 2007].

The first author was privileged to collect more millipedes in 2008 and 2009, being restricted only to the main three islands of the State of Comoros, as part of a FRB programme on the Biodiversity inventory of the Indian Ocean islands, entitled "Connaître pour conserver le patrimoine naturel caché des Comores". Some specimens were also collected by colleagues participating in this programme (referred to in brackets).

This collection which comprises several new records and thus being interesting to publish is fully housed in the Muséum national d'Histoire naturelle in Paris.

A faunistic list

Order Spirobolida

Family Pseudospirobolellidae

Pseudospirobolellus avernus (Butler, 1876)

MATERIAL. 1 juv.: Grande Comore, NG19, Mvouni, belvedere trail plantations $(430 \mathrm{~m}), 28.10 .2008 ; 2 \sigma^{\top} \sigma^{7}, 1$ juv.: Mohéli,
MW05, Lake Dziani Boundouni, degraded dry forest $(60 \mathrm{~m})$, 31.10.2008.; 1 juv.: Mohéli, MW16, Chalet St Antoine, natural forest on crest $(700 \mathrm{~m}), 4.11 .2008$.

REMARKS. This pantropical species is quite common on all of the islands of the Comoro Archipelago [VandenSpiegel \& Golovatch, 2007].

Family Pachybolidae

Leptogoniulus sorornus (Butler, 1876)

MATERIAL. 1 juv.: Anjouan, ND16, Hajoho Bay, back beach, degraded forest (2 m), 16.11.2010; 1 \%: Grande Comore, NG01, Mt Dima Kora, Lake Hantsangoma, banana plants $(1000 \mathrm{~m})$, 19.10.2008.

REMARKS. This pantropical species is quite common on all of the islands of the Comoro Archipelago [VandenSpiegel \& Golovatch, 2007].

\section{Dactylobolus bivirgatus (Karsch, 1881)}

MATERIAL. 1 \%, 1 ㅇ, 1 juv.: Anjouan, ND15, Hajoho Bay, degraded dry coastal environment (15 m), 16.11.2009; 1 + 3 juv.: Anjouan, ND16, Hajoho Bay, back beach, degraded forest $(2 \mathrm{~m})$, 16.11.2009; 1 juv.: Grande Comore, NG10, Mt Karthala, BoboniConvalescence trail, primary forest $(1600 \mathrm{~m}), 24.10 .2008$; 3 juv.: Grande Comore, NG33, Niamaoui Salt Lake, dry and degraded forest $(70 \mathrm{~m}), 11.11 .2008$ (Muratov leg.); 1 ㅇ: Grande Comore, NG35, Niamaoui Salt Lake, degraded forest $(10 \mathrm{~m}), 7.11 .2009$ (Fontaine leg.); 1 juv.: Mohéli, MW01, Itsamia, butte of Hamada Ali, degraded dry forest on slope $(30 \mathrm{~m}), 30.10 .2008$; 1 juv.: Mohéli, MW07, Itsamia, Mtrouni Valley, degraded dry forest $(20 \mathrm{~m})$, 1.11.2008; 2 juv.: Mohéli, MW16, Chalet St Antoine, natural forest on crest $(700 \mathrm{~m}), 4.11 .2008$.

REMARKS. This species has heretofore been found only in the Seychelles, Madagascar and the Comoros, being quite common on all of the islands of the latter archipelago [VandenSpiegel \& Golovatch, 2007]. It can be referred to as a subendemic species. 
Order Spirostreptida

Family Cambalopsidae

Hypocambala anguina (Attems, 1900)

MATERIAL. $1 \sigma^{7}, 1$ : 1 : Anjouan, ND05, top of Mt Ntingui, natural forest (1595 m), 12.11.2009; 1 \%: Grande Comore, NG12, Mt Karthala, degraded forest, SE of Concalescence $(1780 \mathrm{~m})$, 24.10.2008.

REMARKS. In the Comoros, this pantropical species has hitherto been encountered only on Grande Comore [VandenSpiegel \& Golovatch, 2007], thus being new to the fauna of Anjouan.

\section{Family Spirostreptidae}

Seychelleptus variabilis VandenSpiegel \& Golovatch, 2007

MATERIAL. $1 O^{\top}, 1$ P: Anjouan, ND10, Mt Ntingui, natural crest forest under rock ledge (1220 m), 13.11.2009; 1 juv., Mohéli, MW05, Lake Dziani Boundouni, NE shore, degraded dry forest (60 m), 31.10.2008; $10^{7}, 2$ 우: Mohéli, MW17, Chalet St Antoine, $\mathrm{N}$ slope, natural rainforest $(650 \mathrm{~m}), 5.11 .2008$.

REMARKS. This species seems to be endemic to the Comoros, having already been encountered on Anjouan and Mohéli islands, as well as on Mayotte [VandenSpiegel \& Golovatch, 2007].

\section{Charactopygus voeltzkowi (Attems, 1910)}

MATERIAL. 3 우, 1 juv.: Anjouan, ND14, Mro oua Bouénidouari Valley, synanthropic vegetation $(60 \mathrm{~m}), 15.11 .2009$; 2 juv.: Anjouan, ND19, N Col Moya, degraded forest $(1100 \mathrm{~m})$, 17.11.2009; 2 juv.: Grande Comore, NG01, Mt Dima Kora, Lake Hantsangoma, banana plants in degraded forest $(1000 \mathrm{~m})$, 19.10.2008; 2 juv.: Grande Comore, NG17, Mt Karthala, MvouniConvalescence trail, degraded forest $(1000 \mathrm{~m}), 25.10 .2008$; 1 juv.: Grande Comore, NG22, S of Ikoni, Napa Beau, back mangrove (5 m), 29.10.2008; 2 juv.: Grande Comore, NG26, Oussoudjou, Grille Massif, degraded forest (1000 m), 9.11.2008 (Muratov leg.); 1 juv.: Grande Comore, NG30, Tsinimouapanga, degraded forest (985 m), 10.11.2008 (Muratov leg.); 1 †, 1 juv.: Grande Comore, NG35, Niamaoui, Salt Lake, degraded forest (10 m), 7.11.2009 (Fontaine leg.); 2 우: Grande Comore, NG41, SE of Dibouani, Gadjou-Lamdjahala, natural vegetation (500 m), 22.11.2009 (Fontaine leg.); 3 +o: Mohéli, MW03, Itsamia, Boundouni lake, SE of Moutrou, degraded dry forest (20 m), 31.10.2008; 1 juv.: Mohéli, MW05, Dziani Boundouni Lake, NE shore, degraded dry forest (60 m), 31.10.2008; 1 juv.: Mohéli, MW07, NW Itsamia, Mtrouni Valley, degraded dry forest $(20 \mathrm{~m}), 1.11 .2008 ; 9$ juv.: Mohéli, MW08, NW Itsamia, Mtrouni Valley, degraded dry forest $(50 \mathrm{~m})$, 1.11.2008; $10^{\top}, 2$ 우, 1 juv.: Mohéli, MW09, NE of Ouallah, Améré Valley, dense natural forest ( $250 \mathrm{~m}), 2.11 .2008$.

REMARKS. This species seems to be endemic to the Comoros, having already been encountered on all of the islands of the archipelago [VandenSpiegel \& Golovatch, 2007].
Order Polydesmida

Family Paradoxosomatidae

Cnemodesmella comoroensis VandenSpiegel \& Golovatch, 2007

MATERIAL. $1 \sigma^{7}$ : Grande Comore, NG01, E of Mt Dima Kora, Hantsangoma Lake, banana plants (1000 m), 19.10.2008; 1 O: Mohéli, MW10, NE of Ouallah, Améré Valley, Kidogobassi, degraded forest near plantations (160 m), 2.11.2008; 3 우: Mohéli, MW14, Miringoni trail, Chalet St Antoine, natural rainforest $(620$ m) , 4.11.2008; 1 ऽ', 1 O: Mohéli, MW15, Chalet St Antoine, $\mathrm{NE}$ natural forest on crest $(680 \mathrm{~m}), 4.11 .2008 ; 2 \sigma^{\top} \sigma^{\top} 2$ +o : Mohéli, MW17, Chalet St Antoine, N slope, natural rainforest $(650 \mathrm{~m})$, 4.11.2008.

REMARKS. This species seems to be endemic to the Comoros, having been described from Mohéli Island alone [VandenSpiegel \& Golovatch, 2007]. The above is its first record on Grande Comore Island.

\section{Oxidus gracilis (C.L. Koch, 1847)}

MATERIAL. $1 \sigma^{\top}, 2$ 우: Anjouan, ND04, Lake Dzialandée, degraded forest $(900 \mathrm{~m}), 11.11 .2009 ; 1 \mathrm{O}^{7}$ : Anjouan, ND05, top of Mt Ntingui, natural forest (1595 m), 12.11.2009; $19 \sigma^{7} \sigma^{7}, 22$ 우, 7 juv.: Anjouan, ND07, Mt Ntingui, NW crest, natural fern vegetation $(1400 \mathrm{~m}), 12.11 .2009 ; 5 \sigma^{7} \sigma^{\top}, 6$ 우, 3 juv.: Anjouan, ND08, Mt Ntingui, SE crest, natural forest $(1230 \mathrm{~m}), 13.11 .2009 ; 1 \sigma^{7}$, 5 우, 1 juv.: Anjouan, ND09, Mt Ntingui, SE crest, open area in natural forest $(1220 \mathrm{~m}), 13.11 .2009 ; 2$ 90: Anjouan, ND11, Dzialandée Lake, plantations $(920 \mathrm{~m}), 14.11 .2009 ; 1 \sigma^{\top}, 1$ ㅇ, 1 juv.: Anjouan, ND12, trail Dzialandée Lake-Dindi road, Mchakojou, degraded forest $(900 \mathrm{~m}), 14.11 .2009 ; 1$ 9: Anjouan, ND16, Hajoho Bay, back beach, degraded forest ( $2 \mathrm{~m}), 16.11 .2009 ; 1$ q, 8 juv.: Anjouan, ND19, N Col Moya, degraded forest $(1100 \mathrm{~m})$, 17.11.2009; 1 ऽ, 1 ㅇ: Grande Comore, NG07, Mt Karthala, trail Convalescence-Karthala, natural vegetation (1940 m), 23.10.2008; $5 \bigcirc^{\top} \sigma^{\top}, 3$ 우: Grande Comore, NG07bis, Mt Karthala, trail Convalescence-Karthala, natural vegetation (1940 m), 23.10.2008; 1 juv.: Grande Comore, NG08, Mt Karthala, trail Convalescence-Karthala, natural fern vegetation $(1880 \mathrm{~m}), 23.10 .2008 ; 5 \sigma^{7} \sigma^{7}, 10$ 우, 5 juv.: Grande Comore, NG09, Mt Karthala, Boboni trail, primary rainforest $(1490 \mathrm{~m}), 24.10 .2008 ; 1$ ऽ, 13 juv.: Grande Comore, NG10, Mt Karthala, Boboni-Convalescence trail, primary forest (1600 m), 24.10.2008; 5 juv.: Grande Comore, NG11, Mt Karthala, under Convalescence, degraded forest $(1750 \mathrm{~m}), 24.10 .2008$; 8 juv.: Grande Comore, NG12, Mt Karthala, SE of Convalescence, degraded forest $(1780 \mathrm{~m}), 24.10 .2008 ; 2 \sigma^{\top} \sigma^{\top}, 1$ ㅇ, 1 juv.: Grande Comore, NG15, Mt Karthala, Convalescence-Mvouni trail, degraded dry forest $(1450 \mathrm{~m}), 25.10 .2008 ; 1$ O : Grande Comore, NG16, Mt Karthala, Convalescence-Mvouni trail, natural vegetation $(1200 \mathrm{~m}), 25.10 .2008$; 1 juv.: Grande Comore, NG19, Mvouni, belvedere trail, plantations $(430 \mathrm{~m}), 28.10 .2008$; $1 \sigma^{7}$ : Grande Comore, NG20, Mvouni, University campus, ruderal vegetation, rubble $(410 \mathrm{~m}), 28.10 .2008 ; 1 \Im^{7}, 1$ \%: Grande Comore, NG30, S Tsinimouapanga, degraded forest $(985 \mathrm{~m}), 10.11 .2008$ (Muratov leg.); 1 q: Mohéli, MW04, Dziani Boundouni Lake, dry natural forest $(60 \mathrm{~m}), 31.10 .2008 ; 5 \sigma^{7} \sigma^{7}, 5$ +o, 4 juv.: Mohéli, MW09, NE of Ouallah, Améré Valley, Kidogobassi, natural dense forest $(250 \mathrm{~m}), 2.11 .2008$.

REMARKS. This nearly ubiquitous species is one of the most common millipedes in the Comoros [VandenSpiegel \& Golovatch, 2007].

\section{Orthomorpha coarctata (DeSaussure, 1860)}

MATERIAL. 1 ㅇ, 1 juv.: Grande Comore, NG19, Mvouni, belvedere trail, plantations $(430 \mathrm{~m}), 28.10 .2008 ; 1 \sigma^{7}$ : Grande Co- 
more, NG20, Mvouni, University campus, ruderal vegetation, rubble (410 m), 28.10.2008; 1 ○', 1 क: Mohéli, MW01, Itsamia, butte of Hamada Ali, degraded dry forest on slope $(30 \mathrm{~m}), 30.10 .2008$; $6 \sigma^{7} \sigma^{7}, 1$ ㅇ: Mohéli, MW07, NW of Itsamia, Mvouni Valley, degraded dry forest $(20 \mathrm{~m}), 1.11 .2008 ; 14 \sigma^{7} \sigma^{7}, 5$ 우: Mohéli, MW08, NW of Itsamia, Mtrouni Valley, rocky valley in degraded dry forest (50 m), 1.11.2008; 1 juv.: Mohéli, MW17, E of Chalet St Antoine, $\mathrm{N}$ slope, natural rainforest $(650 \mathrm{~m}), 5.11 .2008$

REMARKS. This pantropical species is one of the most common millipedes in the Comoros [VandenSpiegel \& Golovatch, 2007].

\section{Conclusions}

Even though the above small collection contains only nine of the 16 diplopod species known to occur in the Comoros, it includes two new island records: the presumed endemic Cnemodesmella comoroensis on Grande Comore and the pantropical Hypocambala anguina on Anjouan. In general, the fauna of the Comoros is confirmed to be depauperate and dominated by anthropochore elements, but there are a few endemic (4) or subendemic (3) species as well [VandenSpiegel \& Golovatch, 2007].

\section{Reference}

VandenSpiegel D., Golovatch S.I. 2007. The millipedes of the Comoro Islands (Myriapoda: Diplopoda) // Journal of Afrotropical Zoology. Vol.3. P.41-57.

Responsible editor K.G. Mikhailov 